\section{The ALS bucket brigade}

\section{By Benjamin Boettner, Senior Writer}

Now that the ice has thawed from the ALS ice bucket challenge, the question is how to spend the more than $\$ 100$ million donated to The ALS Association after the campaign went viral this summer. Last month, the association answered with plans to use the first \$21.7 million to seed 4 research alliances that will identify biomarkers, create patient cell repositories, accelerate clinical programs and mine the genomes of patients with ALS for new genetic associations and targets.

In ALS (amyotrophic lateral sclerosis), progressive degeneration of motor neurons leads to complete loss of muscle control and paralysis within three years of symptom onset. The only marketed treatment for the disease is Rilutek riluzole from Sanofi and Covis Pharma that slows damage to motor neurons.

The total of $\$ 115$ million raised by the association dwarfs the $\$ 37$ million committed

"The big genetic mutations in
the heritable forms have been
found. Now we need a big
data approach to sequence
genomes from patients with
familial and sporadic ALS to
find the remaining rare and
low-frequency mutations."
-Lucie Bruijn, The ALS Association

\section{New ALS pals} associated genes. S.a.r.l., a small molecule glutamate antagonist

\begin{abstract}
Center, the University of California, San Diego, the Gladstone Institutes, Biogen Idec Inc. and Isis Pharmaceuticals Inc.

The New York Genome Center (NYGC) will receive \$2.5 million from the association in addition to a matching $\$ 2.5$ million from The Tow Foundation for its Consortium of Neurodegenerative Disease. The ALS Association will use another $\$ 1$ million to help create Project MinE, a patient-driven European sequencing initiative coordinated by The Netherlands ALS Center at the University Medical Center Utrecht.

The remaining \$3.2 million will fund treatment centers of excellence across the U.S. that have been certified by the ALS Association and projects to guide companies in navigating the regulatory landscape.
\end{abstract}

Because genetic mutations play a dominant role in the disease pathology of ALS, three of the new alliances-Neuro Collaborative, the NYGC consortium and Project MinE-focus on targeting or finding ALS-

A group of eight major genes cause familial ALS, which accounts for about $10 \%$ of all cases. However, although the same genes are also affected in sporadic forms of the disease, the proportion of unidentified genetic lesions is considerably higher in patients with sporadic ALS (89\%) than in those with familial ALS (32\%). ${ }^{1}$ The two most well-characterized genes in hereditary ALS are superoxide dismutase 1 (SOD1) and chromosome 9 open reading frame 72 (C9orf72).

Neuro Collaborative aims to support the development of antisense therapies that target SOD1 and C9orf72. Preclinical work-largely to ALS-related projects in 2013 by the NIH's National Institute of Neurological Disorders and Stroke and has injected both money and attention into the relatively unstudied disease.

A core part of the ALS Association's strategy for advancing research and creating therapies is the establishment of public-private partnerships with corporate, government, university or other not-forprofit organizations. The association is sticking with that approach for its commitments of the ice bucket money and hopes the resulting new collaborations in the U.S. and Europe will operate synergistically.

"This is only the initial stage of a much larger program that is going to unfold in the next two to three years," the ALS Association's chief scientist, Lucie Bruijn, told SciBX. "Other parts are going to follow. The four collaborations are the initial feeding programs that should lay out the groundwork for major alliances, and there will be more opportunities for solicitation of additional programs."

The largest portion of the announced funding-\$10 million-will go to create ALS Accelerated Therapeutics (ALS-ACT), an academicindustry partnership between General Electric Co.'s GE Healthcare unit and researchers at several universities. A matching gift of $\$ 10$ million to ALS-ACT was donated by the ALS Finding a Cure Foundation.

The association also committed $\$ 5$ million to create Neuro Collaborative, an alliance that includes the Cedars-Sinai Medical by University of California, San Diego's Don Cleveland-established a transgenic mouse model expressing mutant human SOD1 that is one of the dominant preclinical models of the disease. In addition, Cleveland pioneered an antisense oligonucleotide approach for SOD $1 .{ }^{2,3}$ Cleveland is a principal investigator in the alliance.

Bruijn said that the ALS Association funded the Cleveland lab's work in co-developing an antisense oligonucleotide against SOD1 with Isis and an antisense oligonucleotide against C9orf72 with Isis and Biogen Idec. ${ }^{4}$ "Our goal in this area really is to link academic science closer with industry science to promote and derisk these clinical approaches," she said.

Bruijn added that Neuro Collaborative will fund research to further support the development of antisense therapies targeting C90rf72 and gene therapy approaches to correct SOD1 mutations. It will also establish a repository of clinical-grade induced pluripotent stem (iPS) cell lineages in cooperation with researchers at Cedars-Sinai, the University of California, San Francisco School of Medicine and the Gladstone Institutes.

The NYGC's Consortium of Neurodegenerative Disease will use its new funding to perform genomewide association studies using nextgeneration sequencing technology and bioinformatics to identify new genetic associations in ALS.

"The big genetic mutations in the heritable forms have been found," she said. "Now we need a big data approach to sequence genomes from patients 


$$
\begin{aligned}
& \text { "Our goal in this } \\
& \text { area really is to link } \\
& \text { academic science } \\
& \text { closer with industry } \\
& \text { science to promote } \\
& \text { and derisk these } \\
& \text { clinical approaches." } \\
& \text { - Lucie Bruijn, } \\
& \text { The ALS Association }
\end{aligned}
$$

with familial and sporadic ALS to find the remaining rare and lowfrequency mutations."

The NYGC consortium will also synergize with Project MinE, a patient-driven project to mine the genomes of at least 15,000 individuals diagnosed with ALS and 7,500 control individuals for ALS-associated genes.

"From a sequencing perspective, it will be important to analyze ALS by global genetics because of the different prevalence of mutations in different segments of the population," said Bruijn. "This has been clearly seen with C9orf72 mutations. Once genes are identified, they can be looked at in much larger populations represented in the sequence repositories that are going to be built."

The fourth initiative, ALS-ACT, will focus on the development of biomarkers and neuroimaging tools that can support future clinical trials in ALS. In addition, ALS-ACT will fund research to further characterize the roles of the unfolded protein response and neuroinflammatory processes in the disease because identifying molecular targets that could prevent the accumulation of misfolded proteins in ALS neurons or neuroinflammation could lead to compounds that slow neuronal degeneration.

Bruijn also expects cross-pollination between ALS-ACT and the NYGC project. "ALS-ACT is establishing a biobank of ALS cell lines generated from affected patient neurons which can be investigated for biochemical activities," she said. "In the future, the vast data sets emerging from the NYGC's sequencing efforts can be integrated to find interdependencies. It is clear that multiple factors can determine disease onset and severity."

Bruijn told SciBX that details about further funding areas and initiatives will be announced in November.

Boettner, B. SciBX 7(42); doi:10.1038/scibx.2014.1224

Published online Oct. 30, 2014

\section{REFERENCES}

1. Renton, A.E. et al. Nat. Neurosci. 17, 17-23 (2014)

2. Boillée, S. et al. Science 312, 1389-1392 (2006)

3. Smith, R.A. et al. J. Clin. Invest. 116, 2290-2296 (2006)

4. Lagier-Tourenne, C. et al. Proc. Natl. Acad. Sci. USA 110, E4530E4538 (2013)

\section{COMPANIES AND INSTITUTIONS MENTIONED}

The ALS Association, Washington, D.C. ALS Finding a Cure Foundation, no centralized location Biogen Idec Inc. (NASDAQ:BIIB), Weston, Mass. Cedars-Sinai Medical Center, Los Angeles, Calif. Covis Pharma S.a.r.l., Zug, Switzerland General Electric Co. (NYSE:GE), Fairfield, Conn. Gladstone Institutes, San Francisco, Calif. Isis Pharmaceuticals Inc. (NASDAQ:ISIS), Carlsbad, Calif. National Institute of Neurological Disorders and Stroke, Bethesda, Md.

National Institutes of Health, Bethesda, Md.

The Netherlands ALS Center, Utrecht, the Netherlands New York Genome Center, New York, N.Y.

Sanofi (Euronext:SAN; NYSE:SNY), Paris, France

The Tow Foundation, New Canaan, Conn.

University of California, San Diego, La Jolla, Calif.

University of California, San Francisco School of Medicine, San Francisco, Calif.

University Medical Center Utrecht, Utrecht, the Netherlands 